\title{
Enhancing Cycling Stability of Tungsten Oxide Supercapacitor Electrodes via a Boron Cluster-Based Molecular Cross-Linking Approach
}

\author{
Dahee Jung, ${ }^{* a, b}$ Mit Muni, ${ }^{\ddagger a}$ Gustavo Marin, ${ }^{a}$ Roshini Ramachandran, ${ }^{a}$ Maher F. El-Kady, ${ }^{a, b}$ Tanya \\ Balandin, ${ }^{a}$ Richard B. Kaner ${ }^{* a, b, c}$ and Alexander M. Spokoyny ${ }^{* a, b}$

\begin{abstract}
We report our discovery of utilizing perhydroxylated dodecaborate clusters $\left(\left[\mathrm{B}_{12}(\mathrm{OH})_{12}\right]^{2-}\right)$ as a molecular cross-linker to generate a hybrid tungsten oxide material. The reaction of $\left[\mathrm{B}_{12}(\mathrm{OH})_{12}\right]^{2-}$ with $\mathrm{WCl}_{6}$, followed by subsequent annealing of the product at $500{ }^{\circ} \mathrm{C}$ in air successfully produces a tungsten oxide material cross-linked with $B_{12}$-based clusters. The comprehensive structural study of the produced hybrid material confirms a cross-linked network of intact boron-rich clusters and tungsten oxides. We further demonstrate how these robust $\mathrm{B}_{12}$-based clusters in the resulting hybrid tungsten oxide material can effectively preserve the specific capacitance up to 4000 cycles and reduce the charge transfer resistance as well as the response time compared to that of pristine tungsten oxide. Ultimately, this work highlights a promising capability of boron-rich cluster in the hybrid metal oxide to obtain fast and stable supercapacitors with high capacitance.
\end{abstract}

\section{Introduction}

Supercapacitors are a class of energy storage devices found commonly in hybrid electric vehicles, camera components and used as backup power systems. ${ }^{1}$ One class of supercapacitors, called electric double layer capacitors (EDLCs), are a popular option for commercial applications. In EDLCs, electrolyte ions are adsorbed onto the electrode/electrolyte interface of highsurface area carbon and charge is stored solely via this electrostatic double layer. Due to this charge storage process, the structural integrity of the electrode is maintained resulting in high power densities and cycle lifetimes of over one million cycles. ${ }^{2}$ One major drawback of these devices is their low energy density, which limits their use in many applications. To improve the energy density of supercapacitors, many transition metal oxides such as $\mathrm{V}_{2} \mathrm{O}_{5}, \mathrm{Ni}(\mathrm{OH})_{2}, \mathrm{C}_{\mathrm{O}_{3}} \mathrm{O}_{4}, \mathrm{RuO}_{2}, \mathrm{MnO}_{2}$ and $\mathrm{WO}_{3}$ have been investigated. ${ }^{3-8}$ Unlike carbon-based materials, transition metal oxides undergo Faradaic reactions that facilitate charge storage. Due to accessible redox states, transition metal oxides have much higher energy densities, but most suffer from low conductivity and poor cycling stability. ${ }^{9}$ In particular, tungsten oxide has been suggested as a potential alternative for supercapacitor electrode materials due to its redox active properties. ${ }^{8-10}$ Recent efforts have focused on improving tungsten oxide containing supercapacitors including their electrochemical performance, rate, and cycling stability in order to improve this material for energy storage applications. ${ }^{8,10-12}$

Over the past several years, different strategies have been employed to improve the performance of metal oxide-based supercapacitors. One strategy is to switch from bulk materials to nanomaterials (Fig. 1a). ${ }^{7}$ Due to their high surface area, more redox active sites are available on the nanostructured metal oxides; however, the transfer of electrons through these materials is still not as efficient as desired. The vast majority of metal oxide materials for energy storage applications have focused on using conductive supports for metal oxide nanoparticles to facilitate efficient electron transfer (Fig. 1b). ${ }^{13-}$ ${ }^{16}$ 0-D, 1-D, 2-D and 3-D carbon-based materials such as carbon nanodots, carbon nanotubes, graphene and carbon aerogels are often used due to their high conductivity, large surface area and versatility. ${ }^{13-16}$ Conducting polymers such as polypyrrole and polyaniline are also used, however, they too suffer from limited cyclability (Fig. 1c). ${ }^{17}$ Recently, a covalent cross-linking approach has been introduced to manipulate the properties of metal oxides utilizing a perhydroxylated dodecaborate cluster $\left(\left[\mathrm{B}_{12}(\mathrm{OH})_{12}\right]^{2-}\right.$, referred to as $\left.\mathrm{I}\right)$ as a molecular cross-linker. ${ }^{18}$

In this approach, the $\mathrm{B}_{12}$-based cluster, I, was used as a polyol precursor in a manner similar to how organic alcohols are used in the sol-gel process. ${ }^{19}$ Our previous report demonstrated that the reaction of I with titanium tetraisopropoxide $\left(\mathrm{Ti}\left(\mathrm{O}^{i} \mathrm{Pr}\right)_{4}\right)$, followed by annealing at $500{ }^{\circ} \mathrm{C}$, led to the formation of B-O-Ti linkages in the resulting hybrid materials stemming from intact boron clusters. ${ }^{17}$ It should be noted that in contrast to organic molecules, which normally completely decompose during annealing at high temperature $\left(\sim 500{ }^{\circ} \mathrm{C}\right)$ in air, ${ }^{20}$ the inorganic 


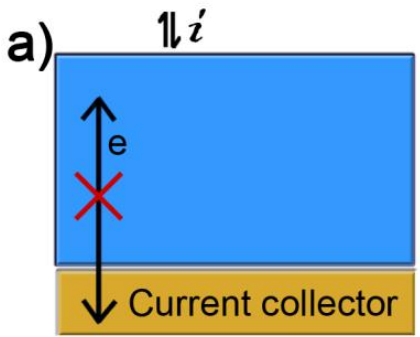

Bulk Metal Oxide

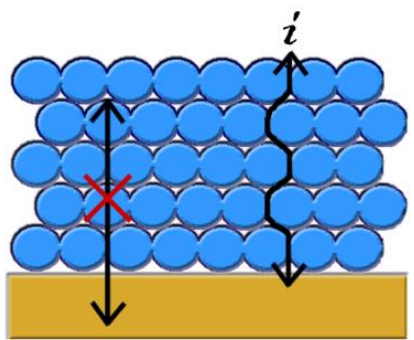

Nanostructured b)

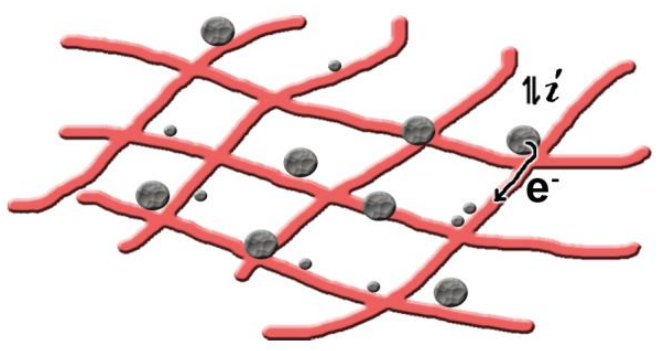

- Metal Oxide Nanoparticle $\sim$ - 0D/1D/2D/3D carbon network c)

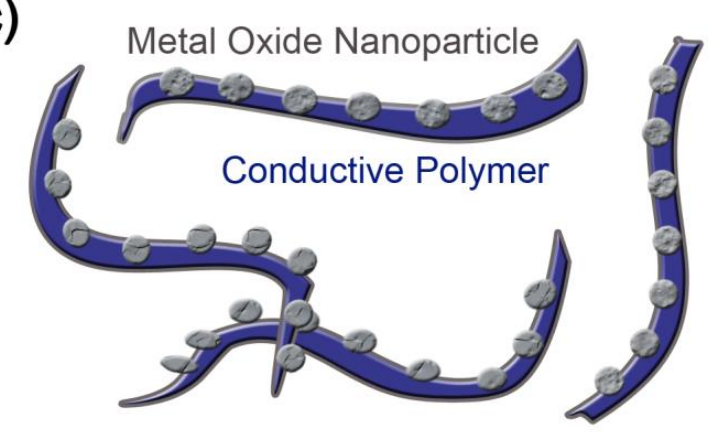

d)

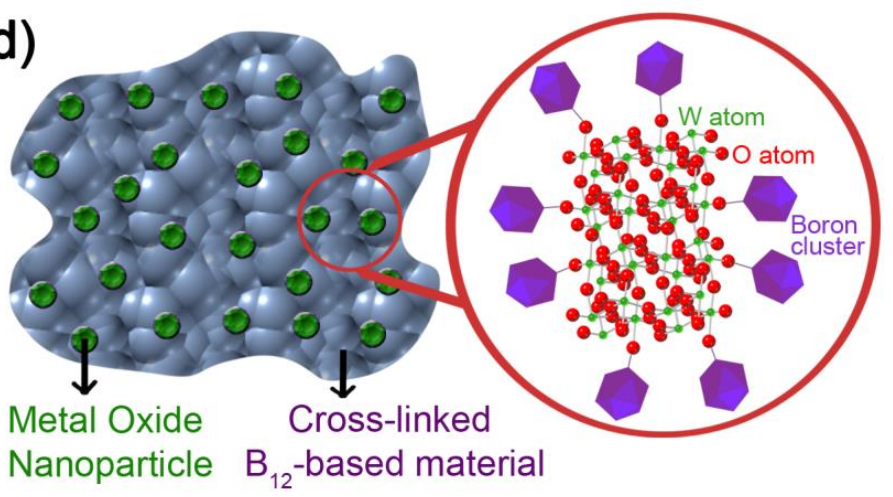

Fig. 1 Overview of existing strategies to improve the performance of metal oxide-based supercapacitors. (a) nanostructuring of bulk metal oxides. (b) carbon composites, and (c) conducting polymer composite materials. (d) Molecularly cross-linked hybrid metal oxides.

polyol, I, remained partially intact allowing it to form a hybrid cross-linked material. The resulting boron-rich hybrid $\mathrm{TiO}_{2}$ material subsequently conferred unique electrochemical properties to the parent metal oxide. Specifically, cross-linking I to the network of $\mathrm{TiO}_{2}$ resulted in increased specific capacitance and a faster electron transfer rate in the resulting hybrid material compared to pristine $\mathrm{TiO}_{2}$. Furthermore, the robust molecular scaffold of $\mathbf{I}$ due to the high degree of electron delocalization provided outstanding thermal and chemical stabilities. ${ }^{21,22}$

In this study, we apply the developed cross-linking approach to a tungsten-based system in order to produce a boron-rich hybrid tungsten oxide material (Fig. 1d). Considering that the molecular cross-linking approach promotes a synergy of the functionalities of the material and cross-linker, ${ }^{23}$ we executed this strategy with tungsten oxide aiming to create a supercapacitor electrode. Our work shows that the incorporation of the robust molecular cross-linker, I, into the tungsten oxide network generates a chemically stable hybrid material, which significantly enhances the long-term cycling stability in the electrochemical reactions. We further demonstrate that the charge transfer resistance and the response time are significantly reduced in the resulting boronrich hybrid material in comparison to that of pristine tungsten oxide.

\section{Experimental}

Materials: Deuterated solvents were purchased from Cambridge Isotope Laboratories and used as received. The following chemicals were purchased from commercial vendors: tungsten hexachloride (Acrosorganics). $\left[\mathrm{N}^{n} \mathrm{Bu}_{4}\right]^{+}$salts of $\left[\mathrm{B}_{12}(\mathrm{OH})_{12}\right]^{2-}$ were synthesized by previously reported methods. ${ }^{24}$

Synthesis of Material 1: The preparation of the microwave reaction was carried out in an inert atmosphere dry-box. In a 10 $\mathrm{ml}$ glass microwave vial, $\mathrm{WCl}_{6}(24 \mathrm{mg}, 0.0611 \mathrm{mmol})$ in acetonitrile $(1 \mathrm{ml})$ was added to a stirring suspension of $\left[\mathrm{N}^{n} \mathrm{Bu}_{4}\right]_{2}\left[\mathrm{~B}_{12}(\mathrm{OH})_{12}\right](50 \mathrm{mg}, 0.0611 \mathrm{mmol})$ in acetonitrile $(0.5$ $\mathrm{ml}$ ). The reaction vial was sealed with a PTFE/silicone cap and then brought outside of the dry-box. The mixture was heated at $120^{\circ} \mathrm{C}$ with stirring in the microwave for $30 \mathrm{~min}$. The red-orange gel reaction mixture was washed with ethanol three times and dried at $80^{\circ} \mathrm{C}$ for $2 \mathrm{~h}$ in air. The dried powder was annealed by heating from room temperature to $500{ }^{\circ} \mathrm{C}$ in air at a rate of 10 ${ }^{\circ} \mathrm{C} / \mathrm{min}$ and holding at $500{ }^{\circ} \mathrm{C}$ for $3 \mathrm{~h}$. Then, the furnace was cooled to room temperature at a rate of $1^{\circ} \mathrm{C} / \mathrm{min}$.

Synthesis of Material 2. In a $10 \mathrm{ml}$ glass microwave vial, $\mathrm{WCl}_{6}$ (290 $\mathrm{mg}, 0.733 \mathrm{mmol}$ ) in acetonitrile $(2 \mathrm{ml})$ was added to a stirring suspension of $\left[\mathrm{N}^{n} \mathrm{Bu}_{4}\right]_{2}\left[\mathrm{~B}_{12}(\mathrm{OH})_{12}\right](50 \mathrm{mg}, 0.0611 \mathrm{mmol})$ in acetonitrile $(0.5 \mathrm{ml})$. The rest of the procedure is the same as that used for material 1.

Synthesis of monoclinic tungsten oxides: In a $10 \mathrm{ml}$ glass microwave vial, $\mathrm{WCl}_{6}(200 \mathrm{mg}, 0.504 \mathrm{mmol})$ was added into ethanol $(3 \mathrm{ml})$. The vial was sealed and transferred to a benchtop microwave reactor. The mixture was heated at $120^{\circ} \mathrm{C}$ with stirring in the microwave for $30 \mathrm{~min}$. After the reaction, the 
product was washed with ethanol 3 times and dried at $80^{\circ} \mathrm{C}$ inair for $2 \mathrm{~h}$. The dried powder was annealed in the same way as materials $\mathbf{1}$ and $\mathbf{2}$.

\section{Characterization Methods and Instrumentation:}

Powder X-ray diffraction (PXRD) patterns were collected on a Panalytical X'Pert Pro X-ray Powder Diffractometer with Cu-Ka radiation. Diffraction spectra were collected from a $2 \theta$ angle of 10 to 80 degree with a step size of 0.04 degree at a rate of 1 degree/min. High-resolution transmission electron microscopy (HRTEM) was performed using a FEI Titan S/TEM operated at $300 \mathrm{kV}$. X-ray photoelectron spectroscopy (XPS) spectra were obtained on an AXIS Ultra DLD instrument (Kratos Analytical Inc., Chestnut Ridge, NY, USA). All XPS spectra were obtained using a monochromatic Al K $\alpha$ X-ray source (12 mA for both survey and high-resolution scans, $15 \mathrm{kV}$ ) with a $300 \times 700 \mathrm{~nm}$ oval spot size. The pressure of analyzer chamber was maintained below $1 \times 10^{-8}$ Torr during the measurement. Spectra were collected with $160 \mathrm{eV}$ pass energy for survey spectra and $20 \mathrm{eV}$ for high-resolution spectra of $\mathrm{C} 1 \mathrm{~s}, \mathrm{O} 1 \mathrm{~s}, \mathrm{~B} 1 \mathrm{~s}$, and $\mathrm{W} 4 f$. All XPS peaks were charge referenced to the adventitious carbon $1 s$ signal at $284.6 \mathrm{eV}$. Infrared (IR) was performed using a PerkinElmer Spectrum One instrument equipped with a universal ATR assembly. Scanning electron microscopy (SEM) was undertaken using a field-emission SEM (JEOL JSM 6700F). ${ }^{11} \mathrm{~B}$ solution NMR spectra were recorded in acetonitrile on AV 400 spectrometers in ambient conditions. ${ }^{11} \mathrm{~B}$ solid-state magic angle spinning (MAS) nuclear magnetic resonance (NMR) spectra were acquired on a $600 \mathrm{MHz}$ (14.1 T) Bruker AVANCE III HD spectrometer using a $3.2 \mathrm{~mm}$ HXY MAS probe. The MAS frequency used was $20 \mathrm{kHz}$ for ${ }^{11} \mathrm{~B}$. The ${ }^{11} \mathrm{~B}$ background signal from the MAS probe was suppressed using the EASY sequence. ${ }^{25}$ The ${ }^{11} \mathrm{~B}$ chemical shift was indirectly referenced to $\mathrm{BF}_{3} \mathrm{O}\left(\mathrm{CH}_{2} \mathrm{CH}_{3}\right)_{2}$ in $\mathrm{CDCl}_{3}$ by using $\mathrm{Cs}_{2}\left[\mathrm{~B}_{12}(\mathrm{OH})_{12}\right](\delta=-17.7 \mathrm{ppm}) .{ }^{18}$ The RF field for the ${ }^{11} \mathrm{~B}$ central transition selective pulse was 66 $\mathrm{kHz} . \mathrm{N}_{2}$ isotherms were obtained at $77 \mathrm{~K}$ on a micromeritics Tristar after activation at $120^{\circ} \mathrm{C}$ under vacuum overnight.

\section{Electrochemical Measurements}

Electrochemical measurements were conducted using a Biologic VMP3 electrochemical workstation (VMP3b-10, Science Instruments). All electrochemical measurements were conducted using a three-electrode set-up that utilized an $\mathrm{Ag} / \mathrm{AgCl}$ reference electrode (Basi Inc.) and a platinum foil counter electrode (Fisher Scientific) in $1.0 \mathrm{M} \mathrm{H}_{2} \mathrm{SO}_{4}$. Working electrodes were made by combining the active material $(\mathrm{m}$ $\mathrm{WO}_{3}$, Material 1 or Material 2) with 10 wt\% PVDF binder and 10 wt\% carbon black in $\mathrm{N}$-methylpyrrolidinone. The solution was mixed using a homogenizer and then drop-cast onto a graphite current collector with an area of $1 \mathrm{~cm}^{2}$. A method developed by Nicholson was used to determine the heterogeneous electron transfer rate constant, $k^{0}$, using the equation:

$$
\varphi=\frac{\left(D_{0} / D_{R}\right)^{\alpha / 2} k^{0}}{\left(\pi D_{0} v F / R T\right)^{\frac{1}{2}}}
$$

where $\varphi$ is a dimensionless kinetic parameter related to the peak separation $\left(\Delta E_{p}\right)$. The other variables represent the diffusion coefficients of the oxidized $\left(D_{0}\right)$ and reduced $\left(D_{R}\right)$ species, transfer coefficient $(\alpha)$, heterogeneous electron rate transfer constant $\left(k^{0}\right)$, scan rate $(v)$, Faraday constant $(\mathrm{F})$, ideal gas constant $(\mathrm{R})$ and temperature $(\mathrm{T})$. The redox system used to determine $k^{0}$ was $2.0 \mathrm{mM} \mathrm{K}_{3}\left[\mathrm{Fe}(\mathrm{CN})_{6}\right]$ dissolved in a $0.10 \mathrm{M}$ $\mathrm{KCl}$ solution. The diffusion coefficients for the oxidized and reduced forms of $\left[\mathrm{Fe}(\mathrm{CN})_{6}\right]^{3-/ 4-}$ in $0.10 \mathrm{M} \mathrm{KCl}$ are similar, therefore $\left(D_{0} / D_{R}\right)^{\alpha / 2} \approx 1.26,27$

Gravimetric capacitance $\left.\left(C_{\mathrm{g}}\right)(\mathrm{F} / \mathrm{g})\right)$ was calculated from the $\mathrm{CV}$ curve of the cathodic scan of each material, using the formula:

$$
C_{g}=\frac{\int_{V_{i}}^{V_{f}} i(V) d V}{m v\left(V_{f}-V_{i}\right)}
$$

where $i$ is the current response, $V_{f}$ and $V_{i}$ are the final and initial voltage, $v$ is the scan rate, and $m$ is the mass of the electrode. The real and imaginary capacitance was calculated from impedance data using the formulas:

$$
\begin{aligned}
& C^{\prime}(\omega)=\frac{-Z^{\prime \prime}(\omega)}{\omega|Z(\omega)|^{2}} \\
& C^{\prime \prime}(\omega)=\frac{Z^{\prime}(\omega)}{\omega|Z(\omega)|^{2}}
\end{aligned}
$$

where $C^{\prime}(\omega)$ is the real capacitance and $C^{\prime \prime}(\omega)$ is the imaginary capacitance. Other variables include the imaginary impedance $\left(-Z^{\prime \prime}(\omega)\right)$, real impedance $\left(-Z^{\prime}(\omega)\right)$ and the complex impedance $\left(|Z(\omega)|^{2}\right)$ where $|Z(\omega)|^{2}=Z^{\prime 2}+Z^{\prime \prime 2} .28$

\section{Results and discussion}

Similar to our previous work with group 4 transition metal oxides, we expected the $\mathrm{B}-\mathrm{OH}$ moieties in I to be able to react with $\mathrm{WCl}_{6}$, forming $\mathrm{B}-\mathrm{O}-\mathrm{W}$ linkages to produce a cross-linked tungsten oxide hybrid material (Fig. 2a). ${ }^{18,21,29-31}$ To test this hypothesis, I was reacted with one equivalent of $\mathrm{WCl}_{6}$ (1 tungsten metal equivalence per $\mathrm{B}_{12}$-based cluster) in acetonitrile at $120^{\circ} \mathrm{C}$ using a benchtop microwave reactor. After $30 \mathrm{~min}$, the reaction mixture produced a red-orange gel (Fig. S1). The supernatant liquid from the reaction was subject to ${ }^{11} \mathrm{~B}$ solution nuclear magnetic resonance (NMR) spectroscopy to detect unreacted $\mathrm{B}_{12}$-based clusters. The ${ }^{11} \mathrm{~B}$ solution NMR spectrum of the supernatant liquid showed an absence of any boron containing species, suggesting that the majority of the boron cluster precursor is contained in the gel-based material (Fig. S2). The solid residue from the reaction was dried and annealed to form a crystalline black material (referred to herein as material 1) (Fig. $\mathbf{2}$ inset). The structure of the resulting solid material was elucidated by powder X-ray diffraction (PXRD); which showed that material 1 possesses a crystalline structure that can be indexed to the monoclinic phase of $\mathrm{WO}_{3}$ (Fig. 2b). ${ }^{32-}$ 
a)
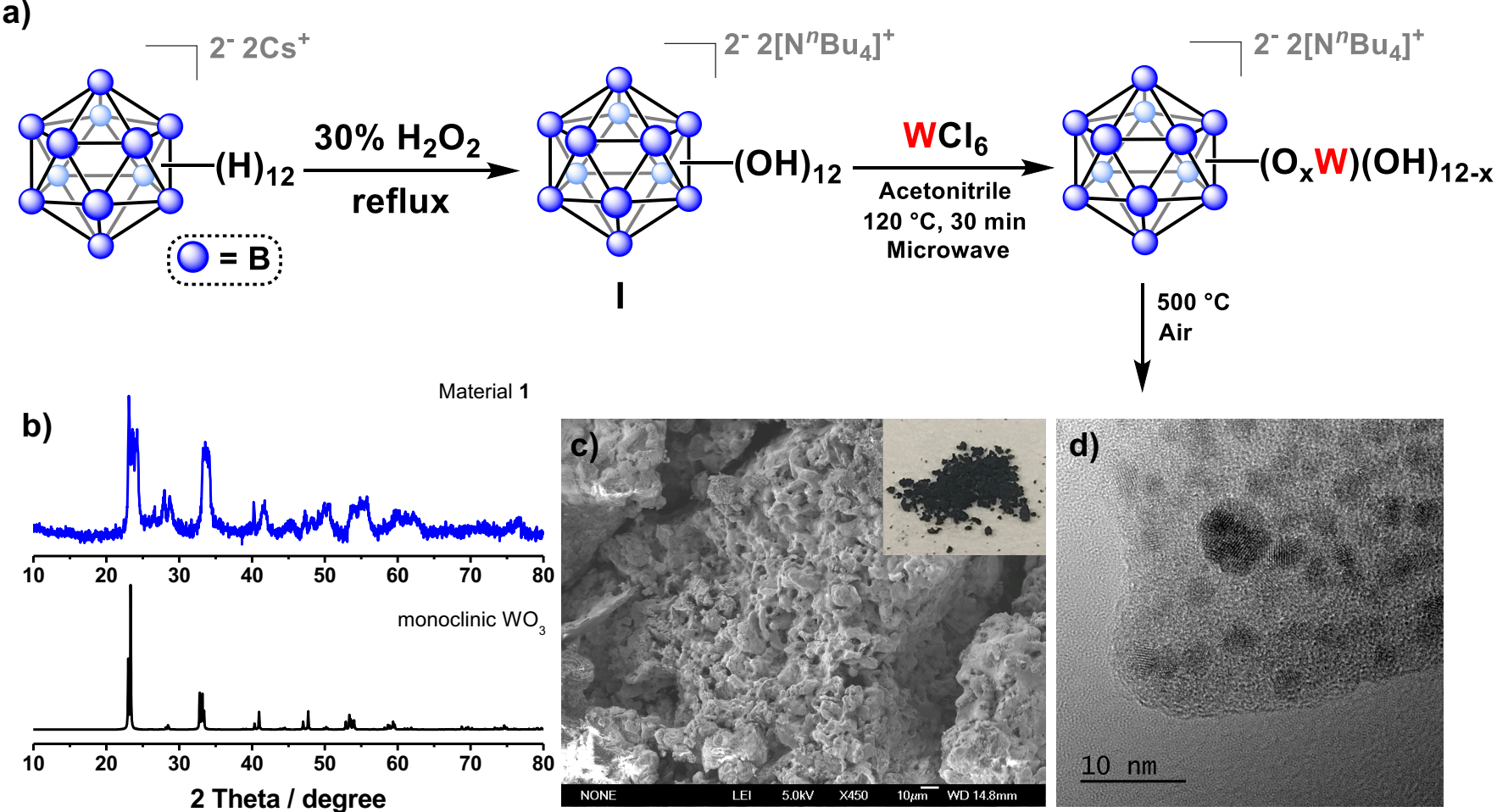

Fig. 2 (a) A synthetic route to produce material 1. (b) powder X-ray diffraction (PXRD) patterns of material 1 and monoclinic $\mathrm{WO}_{3}$. (c) scanning electron microscopy (SEM), (d) transmission electron microscopy (TEM) image of material 1.

34 The broad peaks in the PXRD pattern can be attributed to the small crystal size of $\mathrm{WO}_{3}(<10 \mathrm{~nm})$ in material 1 . The surface morphology and elemental composition of material 1 were obtained using scanning electron microscopy (SEM) and energy dispersive X-ray spectroscopy (EDS). The SEM images of $\mathbf{1}$ suggested the likely formation of a porous structure at the surface upon cross-linking boron-rich clusters to the tungsten oxide network (Fig. 2c). Moreover, the EDS elemental analysis showed that material 1 consisted of boron, oxygen, tungsten and carbon with 12.2, 73.9, 4.6 and $7.5 \%$, respectively (Fig. S3). Noticeably, no chlorine was detected, providing additional support that the B-O-W linkage is possibly formed through the reaction between $\mathrm{I}$ and $\mathrm{WCl}_{6}$ with the concomitant formation of $\mathrm{HCl}$. Furthermore, the Fourier transform infrared (FT-IR) spectrum of material 1 displayed a broad peak below the 1000 $\mathrm{cm}^{-1}$ region which is characteristic of $\mathrm{W}-\mathrm{O}$ stretching vibrations
(Fig. S4). ${ }^{35}$ High-resolution TEM (HRTEM) images of material 1 highlighted the morphology of nanocrystalline $\mathrm{WO}_{3}$ particles with additional amorphous material, which can be attributed to intact boron cluster units and boron oxide (Fig. 2d). X-ray photoelectron spectroscopy (XPS) measurements were performed to investigate the surface environment of material 1. The boron $1 \mathrm{~s}$ region exhibited two components at 189.8 and $194.1 \mathrm{eV}$, corresponding to the presence of intact dodecaborate clusters as well as boron oxide and borates (Fig. 3a). The W $4 \mathrm{f}$ XPS spectrum consisted of two peaks of $4 f_{7 / 2}$ and $4 f_{5 / 2}$ at 36.2 and $38.3 \mathrm{eV}$, respectively which are well-matched to those of $\mathrm{W}(\mathrm{VI})$ trioxides (Fig. 3b). The $\mathrm{O}$ 1s region showed multiple components at 530.8, 531.4, and $533.1 \mathrm{eV}$, which can be assigned to $\mathrm{O}-\mathrm{W}, \mathrm{O}-\mathrm{C}$, and $\mathrm{O}-\mathrm{B}$, respectively (Fig. $\mathbf{3 c}$ ). ${ }^{36}$ The atomic compositions obtained from XPS analyses demonstrated that boron (21\%) and oxygen (51\%) are dominant while there is a)

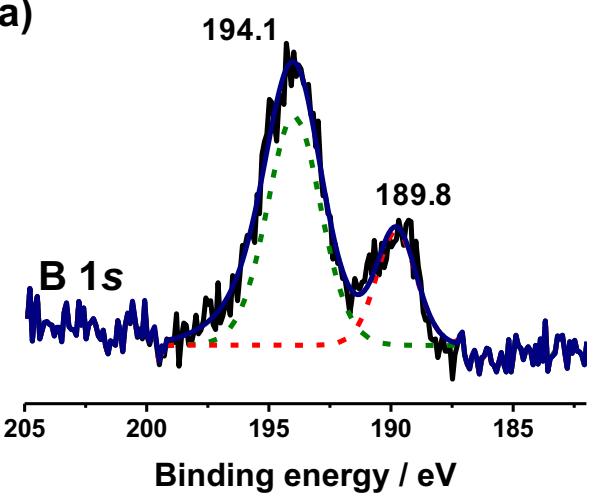

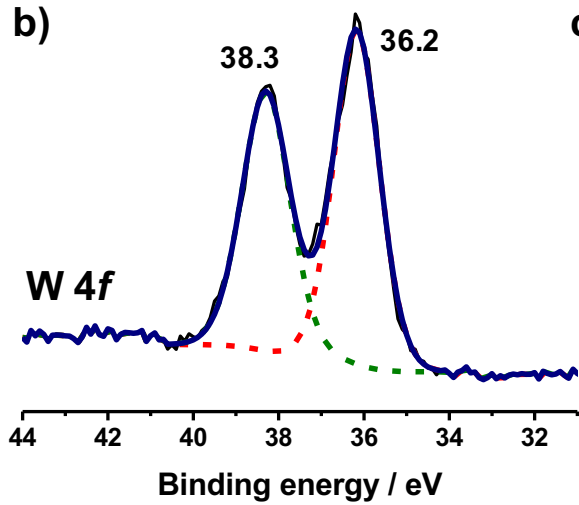

c)

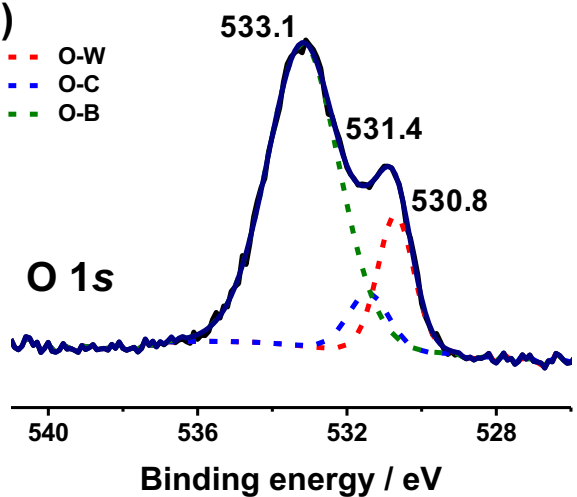

Fig. 3 X-ray photoelectron spectroscopy (XPS) spectra of material 1. (a) B 1s, (b) W $4 f$, (c) $01 s$ region. 
only $5 \%$ of tungsten at the surface (Table S1). This result is consistent with the TEM image of material $\mathbf{1}$ which suggests that the tungsten oxide nanoparticles are embedded in the layer consisting of a cross-linked network of intact $\mathrm{B}_{12}$-based clusters as well as some boron oxides.

The presence of intact $B_{12}$-based clusters in material $\mathbf{1}$ was directly verified using solid-state ${ }^{11} B$ NMR spectroscopy, which can provide the local environment of ${ }^{11} \mathrm{~B}$ atoms in the material. The 1D single-pulse ${ }^{11} \mathrm{~B}$ magic angle spinning (MAS) SSNMR of material 1 showed three distinct features. The peak at -15.7 ppm was assigned to the sub-surface dodecaborate clusters which are directly bound to the tungsten oxide network based on the signal from a reference material containing $\mathrm{Cs}_{2}\left[\mathrm{~B}_{12}(\mathrm{OH})_{12}\right]$ (Fig. S5 and S6). Two additional signals at 0.7 and $12.9 \mathrm{ppm}$ can be attributed to the partially intact $B_{12}$-based clusters at the surface and to boron oxide or borates, respectively. ${ }^{18,37}$ These observations are consistent with our previous work on Ti-based system which showed three distinct signals at $-16,2$, and $17 \mathrm{ppm}$ in the ${ }^{11} \mathrm{~B}$ MAS NMR that can be attributed to the intact $B_{12}$-based clusters, partially intact clusters at the particle surface and boron oxides and borates in the material, respectively. ${ }^{18}$ The combined structural and morphological characterizations of material $\mathbf{1}$ validate that the hybrid tungsten oxide material is composed of nano-crystalline monoclinic $\mathrm{WO}_{3}$ particles cross-linked with molecular boron clusters, I, as represented in Figure 1d.

Many metal oxide-based supercapacitors provide high specific capacitance. Although promising values ranging from $232-510 \mathrm{~F} / \mathrm{g}$ for various morphologies of $\mathrm{WO}_{3}$ have been achieved, these high capacitances are typically limited to very slow scan rates. ${ }^{38,39}$ The charge storage mechanism of $\mathrm{WO}_{3}$ can be rationalized as two stages of proton intercalation reaction accompanied by the change in oxidation state of $\mathrm{W}^{6+}$ to $\mathrm{W}^{5+: 40}$

$$
\begin{aligned}
& \mathrm{WO}_{3-\mathrm{x}}+n \mathrm{H}^{+}+n \mathrm{e}^{-} \leftrightarrow \mathrm{H}_{n} \mathrm{WO}_{3-\mathrm{x}} \\
& \mathrm{H}_{n} \mathrm{WO}_{3-\mathrm{x}}+(1-n) \mathrm{e}^{-}+(1-n) \mathrm{H}^{+} \leftrightarrow \mathrm{HWO}_{3-\mathrm{x}}(0<\mathrm{n}<1)
\end{aligned}
$$

To determine the capacitance of material 1, we conducted cyclic voltammetry at varying scan rates with a voltage window from $-0.5 \mathrm{~V}$ to $0.6 \mathrm{~V}$ (Fig. 4a). As a control experiment, we also prepared monoclinic tungsten oxide $\left(\mathrm{m}-\mathrm{WO}_{3}\right)$ and another hybrid tungsten oxide material using a different ratio of $\mathrm{B}_{12}$ based cluster to tungsten (12-tungsten metal equivalences per $\mathrm{B}_{12}$-based cluster, referred to material 2) to provide benchmark values (Fig. S7-12). For pristine $\mathrm{m}^{-} \mathrm{WO}_{3}$, the maximum capacitance value at $1 \mathrm{mV} / \mathrm{s}$ was determined to be $283 \mathrm{~F} / \mathrm{g}$, which is similar to previously reported values. ${ }^{39}$ For materials 1 and $\mathbf{2}$, the specific capacitances were determined to be $31 \mathrm{~F} / \mathrm{g}$ a)
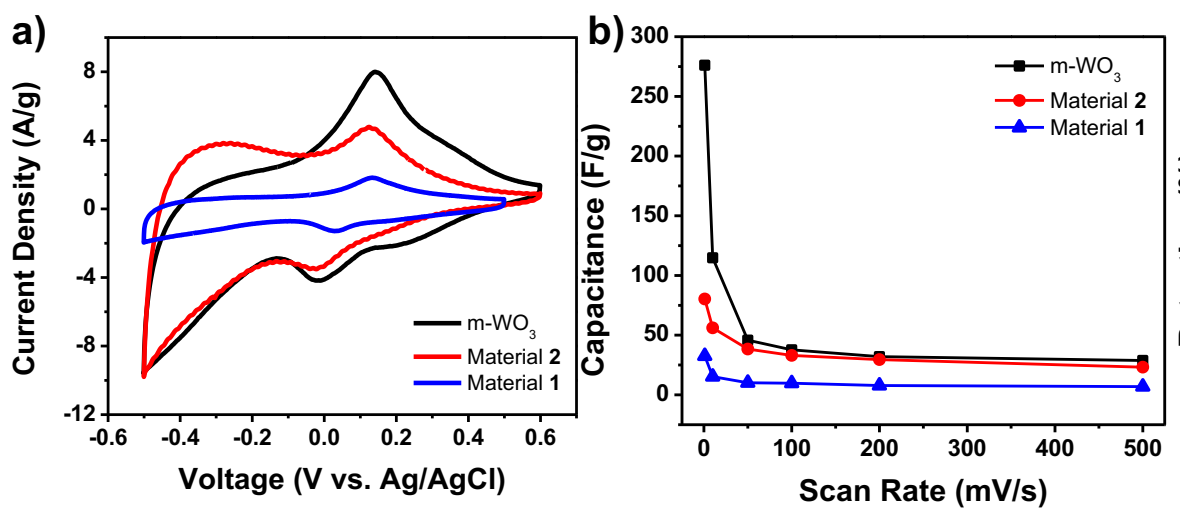

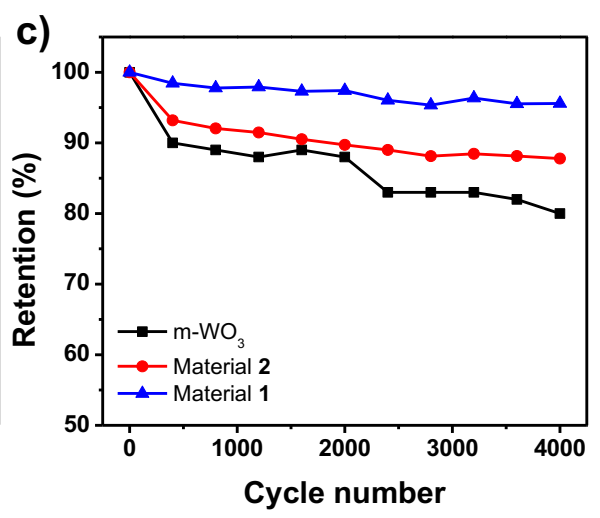

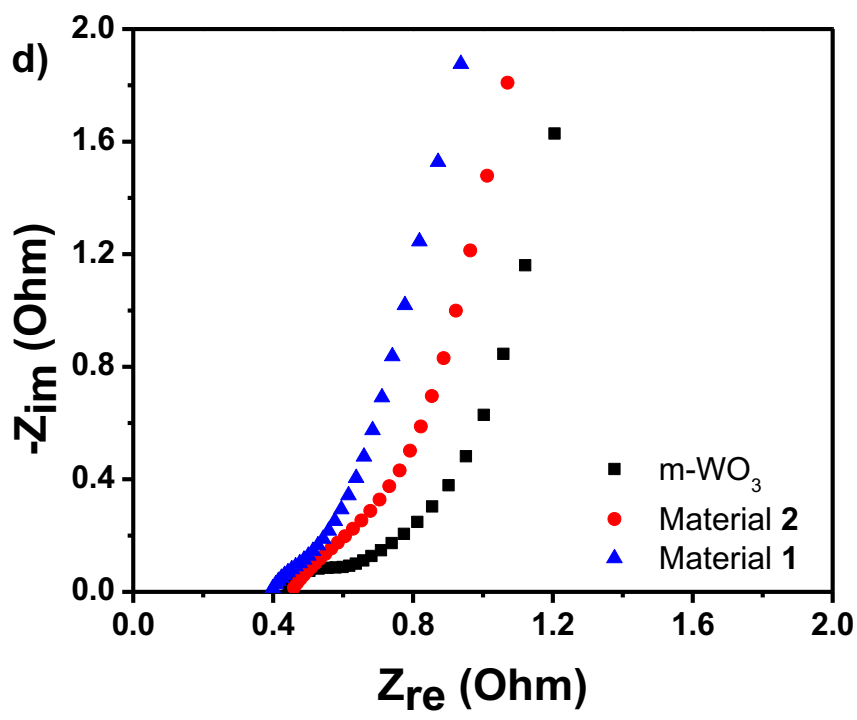

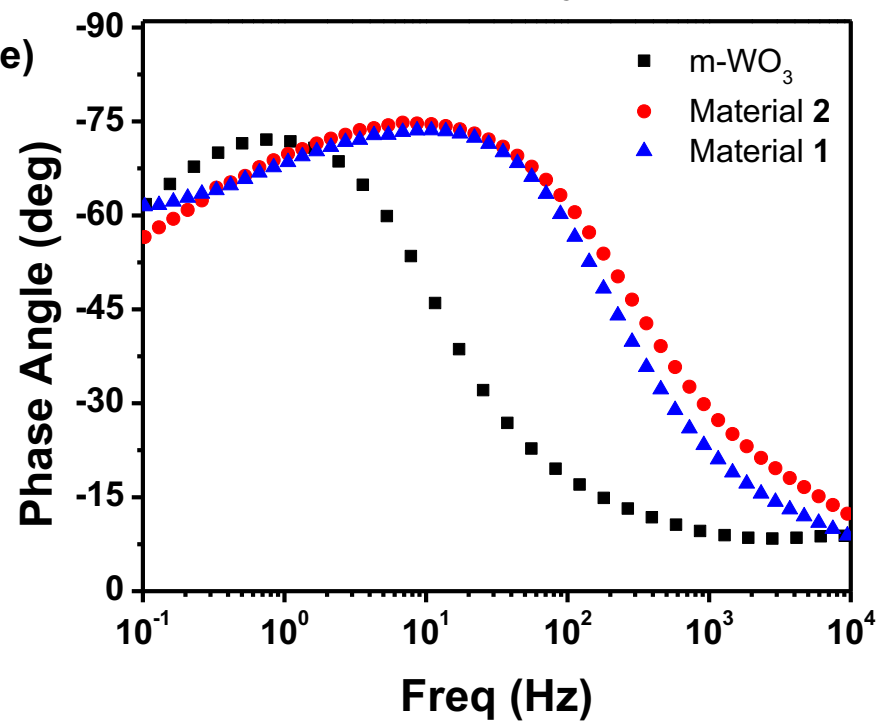

Fig. 4 Electrochemical properties of material 1, 2, and monoclinic $\mathrm{WO}_{3}\left(\mathrm{~m}-\mathrm{WO}_{3}\right)$. (a) cyclic voltammograms at a scan rate of $100 \mathrm{mV} / \mathrm{s}$, (b) specific capacitance as a function of scan rates (c) cycling performance, (d) Nyquist and (e) Bode plots of material 1, 2, and $\mathrm{m}$-WO $\mathrm{W}_{3}$ control. 

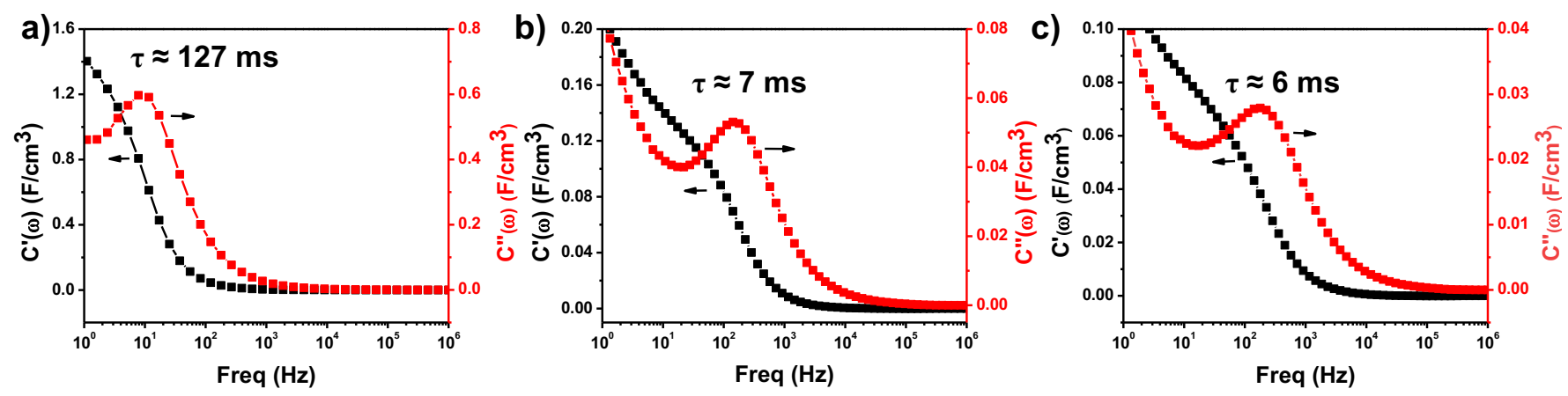

Fig. 5 Real, $C^{\prime}(\omega)$, and imaginary, $C^{\prime \prime}(\omega)$, capacitance as a function of frequency with relaxation times for (a) $m$-WO $\mathrm{W}_{3}$, (b) material $\mathbf{1}$ and $(c)$ material 2.

and $81 \mathrm{~F} / \mathrm{g}$, respectively (Fig. $\mathbf{4 b}$ ). Although there is a large capacitance difference at slow scan rates, the capacitance of material 2 and $\mathrm{m}-\mathrm{WO}_{3}$ are similar at scan rates of $50 \mathrm{mV} / \mathrm{s}$ and greater. Additionally, $\mathrm{m}-\mathrm{WO}_{3}$ loses over $80 \%$ of its initial capacitance at a scan rate of $50 \mathrm{mV} / \mathrm{s}$, whereas the loss in capacitance of material $\mathbf{1}$ and $\mathbf{2}$ exhibit lower rates of capacitance loss. We tentatively attribute the lower capacitance values of material $\mathbf{1}$ and $\mathbf{2}$ to the lower effective surface area available to the electrolyte (Fig. S13).

Due to constant redox reactions, the structure of the metal oxide can become strained, leading to structural distortions during the charge-discharge process. This can cause cracking of the electrode over time, leading to a poor cycle life. ${ }^{41}$ Therefore, the development of metal oxide-based materials that possess high thermal and chemical stability, which eventually affect the cycling stability would be necessary for the practical use of metal oxides as supercapacitor electrodes. To investigate longterm stability, all three materials were charged and discharged 4,000 times at a current density of $5 \mathrm{~A} / \mathrm{g}$. While the pristine $\mathrm{m}-$ $\mathrm{WO}_{3}$ only preserved $80 \%$ of its initial capacitance, material $\mathbf{1}$ and material $\mathbf{2}$ proved to have higher stability with material $\mathbf{2}$ preserving $88 \%$ of its initial capacity and material 1 preserving $96 \%$ of its initial capacity (Fig. 4c). Due to the extensive crosslinking present in materials $\mathbf{1}$ and $\mathbf{2}$, we speculate that the molecular cross-linker I may act as a mechanical support to $\mathrm{WO}_{3}$, preventing structural degradation over time.

Another consequence of the low conductivity in most metal oxides is that both the charge transfer resistance and sheet resistance of the electrode are relatively high. Electrical impedence spectroscopy was used to investigate the resistive properties of the materials. In the Nyqiust plot, both materials $\mathbf{1}$ and $\mathbf{2}$ show a significant decrease in charge transfer resistance compared to pristine $\mathrm{m}-\mathrm{WO}_{3}$ (Fig. 4d). Additionally, in the lower frequency regions, the slope of materials $\mathbf{1}$ and $\mathbf{2}$ are steeper, indicating less diffusion resistance. From the Bode plot, the minimum phase angle is approximately $-75^{\circ}$ for all three samples, however, there is a large shift to higher frequencies for the cross-linked materials indicating a faster relaxation time (Fig. 4e). The relaxation time is an important factor of merit, indicating when a material transitions from resistive to capacitive behavior and is the time required to deliver the stored energy and power efficiently. ${ }^{42}$ To determine the relaxation time of all three materials, we modeled the real, $C^{\prime}(\omega)$, and imaginary, $C^{\prime \prime}(\omega)$, capacitance as a function of frequency (Fig. 5). ${ }^{28} \mathrm{~A}$ remarkable decrease in the response time for both materials $\mathbf{1}$ and $\mathbf{2}$ was observed. Pristine $\mathrm{m}-\mathrm{WO}_{3}$ shows a relaxation time of $127 \mathrm{~ms}$, whereas materials $\mathbf{1}$ and $\mathbf{2}$ have a relaxation time of $7 \mathrm{~ms}$ and $6 \mathrm{~ms}$, respectively. We attribute these results to the increase in conductivity due to the crosslinking present in materials $\mathbf{1}$ and $\mathbf{2}$. Previous reports have shown that the molecular cross-linker, I, has remarkable kinetic stability and displays pseudometallic behavior due to extensive electron delocalization. ${ }^{43-45}$

In order to determine the possible electrocatalytic properties of material 1, we studied its electron-transfer capability by using the ferri/ferrocene redox couple (Fig. S14). The peak-to-peak separation of material $\mathbf{1}$ is smaller than pristine $\mathrm{m}-\mathrm{WO}_{3}$, leading to a $21 \%$ increase in the observed electron-transfer-rate constant, $k^{0}$, with a value of $1.64 \times 10^{-3}$ $\mathrm{cm} \mathrm{s}^{-1}$. The oxidation peak observed at $0.8 \mathrm{~V}$ can be attributed to the formation of the radical species $\left[\mathrm{B}_{12}(\mathrm{OH})_{12}\right]^{1-.46}$

\section{Conclusions}

In summary, we have demonstrated a successful synthesis of a boron-rich hybrid tungsten oxide material and its utilization as a supercapacitor electrode with superior cycling stability to pristine tungsten oxide. The comprehensive structural study of material 1 revealed the formation of a cross-linked network of tungsten oxide nanoparticles with intact molecular boron-rich clusters. Importantly, the incorporation of the robust molecular cross-linker, I, into the network of tungsten oxide effectively preserved the specific capacitance of the material up to 4000 cycles and reduce the charge transfer resistance as well as the response time in comparison to that of pristine $\mathrm{m}-\mathrm{WO}_{3}$. Further improvements such as increasing the effective surface area could allow for capacitance values mirroring pristine $\mathrm{m}-\mathrm{WO}_{3}$, while retaining the benefits of incorporating $\mathbf{I}$. These results highlight the promising capability of I in the hybrid metal oxide materials to ultimately obtain fast and stable metal oxide-based supercapacitors with high capacitances. This work further highlights the potential promise of boron cluster-containing materials as unique building blocks for the next generation of energy storage and conversion materials and devices. ${ }^{47-65}$

\section{Conflicts of interest}


There are no conflicts to declare.

\section{Acknowledgements}

This work was supported by NSF Grant CHE-1846849 (NSF CAREER Award to A.M.S.). A.M.S. is a Research Corporation for Science Advancement (RCSA) Cottrell Scholar and a Dreyfus Foundation Camille Dreyfus Teacher-Scholar Awardee. D. J. acknowledges the UCLA Graduate Division for the Dissertation Year Fellowship. R.B.K. thanks the Dr. Myung Ki Hong Endowed Chair in Materials Innovation at UCLA for support. The authors thank Nicholas A. Bernier for helpful discussions.

\section{Notes and references}

1 P. Simon and Y. Gogotsi, Nat. Mater., 2008, 7, 845-854.

2 A. Noori, M. F. El-Kady, M. S. Rahmanifar, R. B. Kaner and M. F. Mousavi, Chem. Soc. Rev., 2019, 48, 1272-1341.

3 C. Y. Foo, A. Sumboja, D. J. H. Tan, J. Wang and P. S. Lee, Adv. Energy Mater., 2014, 4, 1400236.

4 J. Ji, L. L. Zhang, H. Ji, Y. Li, X. Zhao, X. Bai, X. Fan, F. Zhang and R. S. Ruoff, Nanoporous Ni(OH)2 ACS Nano 2013, 7, 62376243.

5 M. Li, M. F. El-Kady, J. Y. Hwang, M. D. Kowal, K. March, H. Wang, Z. Zhao and R. B. Kaner, Nano Research, 2018, 11, 2836-2846.

6 L. Y. Chen, Y. Hou, J. L. Kang, A. Hirata, T. Fujita and M. W. Chen, Adv. Energy Mater., 2013, 3, 851-856.

7 M. F. El-Kady, M. Ihns, M. Li, J. Y. Hwang, M. F. Mousavi, L. Chaney, A. T. Lech and R. B. Kaner, PNAS, 2015, 112, 42334238.

8 A. K. Nayak, A. K. Das and D. Pradhan, ACS Sustain. Chem. Eng., 2017, 5, 10128-10138.

9 C. Liu, F. Li, M. Lai-Peng and H. M. Cheng, Advanced Materials., 2010, 22, E28-E62.

10 F. Zheng, C. Xi, J. Xu, Y. Yu, W. Yang, P. Hu, Y. Li, Q. Zhen, S. Bashir and J. L. Liu, J. Alloys Compd., 2019, 772, 933-942.

11 X. Liu, G. Sheng, M. Zhong and X. Zhou, Nanoscale, 2018, 10, 4209-4217.

12 M. Qiu, P. Sun, L. Shen, K. Wang, S. Song, X. Yu, S. Tan, C. Zhao and W. Mai, J. Mater. Chem. A, 2016, 4, 7266-7273.

13 L. Hu, W. Chen, X. Xie, N. Liu, Y. Yang, H. Wu, Y. Yao, M. Pasta, H. N. Alshareef and Y. Cui, ACS Nano, 2011, 5, 8904-8913.

14 J. Y. Hwang, M. F. El-Kady, Y. Wang, L. Wang, Y. Shao, K. Marsh, J. M. Ko and R. B. Kaner, Nano Energy, 2015, 18, 57-70.

15 Y. H. Lin, T. Y. Wei, H. C. Chien and S. Y. Lu, Adv. Energy Mater., 2011, 1, 901-907.

16 Y. Zhu, X. Ji, C. Pan, Q. Sun, W. Song, L. Fang, Q. Chen and C. E. Banks, Energy Environ. Sci.,2013, 6, 3665-3675.

17 H. Wei, X. Yan, S. Wu, Z. Luo, S. Wei and Z. Guo, J. Phys. Chem. C, 2012, 116, 25052-25064.

18 D. Jung, L. M. A. Saleh, Z. J. Berkson, M. F. El-Kady, J. Y. Hwang, N. Mohamed, A. I. Wixtrom, E. Titarenko, Y. Shao, K. McCarthy, J. Guo, I. B. Martini, S. Kraemer, E. C. Wegener, P. Saint-Cricq, B. Ruehle, R. R. Langeslay, M. Delferro, J. L. Brosmer, C. H. Hendon, M. Gallagher-Jones, J. Rodriguez, K. W. Chapman, J. T. Miller, X. Duan, R. B. Kaner, J. I. Zink, B. F. Chmelka and A. M. Spokoyny, Nat. Mater., 2018, 17, 341-348.

19 A. E. Danks, S. R. Hall and Z. Schnepp, Mater. Horiz., 2016, 3, 91-112.

20 I. Salzmann and G. Heimel, J. Electron Spectrosc. Relat. Phenom., 2015, 204, 208-222.

21 D. Jung, F. Raffan-Montoya, R. Ramachandran, Y. Zhang, T. Islamoglu, G. Marin, E. A. Qian, R. M. Dziedzic, O. K. Farha, S.
I. Stoliarov and A. M. Spokoyny, Chem. Commun., 2019, 55, 8852-8855.

22 J. L. Barton, A. I. Wixtrom, J. A. Kowalski, E. A. Qian, D. Jung, F. R. Brushett and A. M. Spokoyny, ACS Appl. Energy Mater., 2019, 2, 4907-4913.

23 R. Ramachandran, D. Jung and A. M. Spokoyny, NPG Asia Mater., 2019, 11:19.

24 A. I. Wixtrom, Y. Shao, D. Jung, C. W. Machan, S. N. Kevork, E. A. Qian, J. C. Axtell, S. I. Khan, C. P. Kubiak and A. M. Spokoyny, Inorg. Chem. Front., 2016, 3, 711-717.

25 C. Jaeger and F. Hemmann, Solid State Nucl. Mag., 2014, 57, 22-28.

26 J. Moldenhauer, M. Meier and D. W. Paul, J. Electrochem. Soc, 2016, 163, H672-H678.

27 S. J. Konopka and M. Bruce, Anal. Chem. 1970, 42, 1741-1746.

28 P. L. Taberna, P. Simon and J. F. Fauvarque, J. Electrochem. Soc., 2003, 150, A292-A300.

29 O. K. Farha, R.L. Julius, M. W. Lee, R. E. Huertas, C. B. Knobler and M. F. Hawthorne, J. Am. Chem. Soc., 2005, 127, 18243-18251.

30 M. S. Messina, J. C. Axtell, Y. Wang, P. Chong, A. I. Wixtrom, K. O. Kirlikovali, B. M. Upton, B. M. Hunter, O. S. Shafaat, S. I. Khan, J. R. Winkler, H. B. Gray, A. N. Alexandrova, H. D. Maynard and A. M. Spokoyny, J. Am. Chem. Soc., 2016, 138, 6952-6955.

31 E. A. Qian, A. I. Wixtrom, J. C. Axtell, A. Saebi, D. Jung, P. Rehak, Y. Han, E. H. Moully, D. Mosallaei, S. Chow, M. S. Messina, J. Y. Wang, A. T. Royappa, A. L. Rheingold, H. D. Maynard, P. Král and A. M. Spokoyny, Nat. Chem., 2017, 9, 333-340.

32 A. Jain, S. P. Ong, G. Hautier, W. Chen, W.D. Richards, S. Dacek, S. Cholia, D. Gunter, D. Skinner, G. Ceder and K.A. Persson, APL Materias, 2013, 1, 011002.

33 N. L. Houx, G. Pourroy, F. Camerel, M. Comet and D. Spitzer, J. Phys. Chem. C, 2010, 114, 155-161.

34 C. Chacón, M. Rodríguez-Pérez, G. Oskam and G. RodríguezGattorno, J. Mater. Sci: Mater Electron, 2015, 26, 5526-5531.

35 N. Sharma, M. Deepa, P. Varshney and S. A. Agnihotry, Thin Solid Films, 2001, 401, 45-51.

36 T. G. G. Maffeis, D. Yung, L. LePennec, M. W. Penny, R. J. Cobley, E. Comini, G. Sberveglieri and S. P. Wilks, Surf. Sci., 2007, 601, 4953-4957.

37 N. Feng, A. Zheng, Q. Wang, P. Ren, X. Gao, S.-B. Liu, Z. Shen, T. Chen and F. Deng, J. Phys. Chem. C, 2011, 115, 2709-2719.

38 V. Lokhande, A. Lokhande, G. Namkoong, J. H. Kim and T. Ji, Results Phys., 2019, 12, 2012-2020.

39 W. Sun, M. T. Yeung, A. T. Lech, C. W. Lin, C. Lee, T. Li, X. Duan, J. Zhou and R. B. Kaner, Nano Lett., 2015, 15, 4834-4838.

40 S. Cong, Y. Tian, Q. Li, Z. Zhao and F. Geng, Adv. Mater., 2014, 26, 4260-4267.

41 M. Zhi, C. Xiang, J. Li, M. Li and N. Wu, Nanoscale, 2013, 5, 7288.

42 C. Portet, G. Yushin and Y. Gogotsi, Carbon, 2007, 45, 25112518.

43 M. W. Lee, O. K. Farha, M. F. Hawthorne and C. H. Hansch, Angew. Chem. Int. Ed., 2007, 46, 3018-3022.

44 A. R. Pitochelli and M. F. Hawthorne, J. Am. Chem. Soc., 1960, 82, 3228-3229.

45 R. Hoffmann and W. N. Lipscomb, J. Chem. Phys., 1962, 36, 2179-2189.

46 N. Van, I. Tiritiris, R. F. Winter, B. Sarkar, P. Singh, C. Duboc, A. Muñoz-Castro, R. Arratia-Pérez, W. Kaim and T. Schleid, Chem. Eur. J., 2010, 16, 11242-11245.

47 L. Cerdán, J. Braborec, I. Garcia-Moreno, A. Costela and M. G. S. Londesborough, Nat Commun., 2015, 6, 5958

48 J. A. Teprovich Jr., H. Colón-Mercado, A. L. Washington II, P. A. Ward, S. Greenway, D. M. Missimer, H. Hartman, J. Velten, J. 
H. Christian and R. Zidan, J. Mater. Chem. A, 2015, 3, 2285322859.

49 R. Núñez, M. Tarrés, A. Ferrer-Ugalde, F. F. de Biani and F. Teixidor, Chem. Rev., 2016, 116, 14307-14378.

50 H. Naito, K. Nishino, Y. Morisaki, K. Tanaka and Y. Chujo, J. Mater. Chem. C, 2017, 5, 10047-10054.

51 R. Ruiz-Rosas, I. Fuentes, C. Viñas, F. Teixidor, E. Morallón and D. Cazorla-Amorós, Sustainable Energy Fuels, 2018, 2, 345352.

52 C. -W. Kung, K. Otake, C. T. Buru, S. Goswami, Y. Cui, J. T. Hupp, A. M. Spokoyny and O. K. Farha, J. Am. Chem. Soc., 2018, 140, 3871-3875.

53 N. T. Hahn, T. J. Seguin, K. -C. Lau, C. Liao, B. J. Ingram, K. A. Persson and K. R. Zavadil, J. Am. Chem. Soc., 2018, 140, 35, 11076-11084.

54 M. Kar, O. Tutusaus, D. R. MacFarlane and R. Mohtadi, Energy Environ. Sci., 2019, 12, 566-571.

55 Y. Zhu, S. Gao and N. S. Hosmane, Inorg. Chim. Acta, 2018, 471, 577-586.

56 S. P. Fisher, A. W. Tomich, S. O. Lovera, J. F. Kleinsasser, J. Guo, M. J. Asay, H. M. Nelson and V. Lavallo, Chem. Rev., 2019, 119, 8262-8290.

57 T. J. Aubry, J. C. Axtell, V. M. Basile, K. J. Winchell, J. R. Lindemuth, T. M. Porter, J. - Y. Liu, A. N. Alexandrova, C. P. Kubiak, S. H. Tolber, A. M. Spokoyny and B. J. Schwartz, Adv. Mater., 2019, 31, 1805647.

58 Z. Peng, K. Zhang, Z. Huang, Z. Wang, S. Duttwyler, Y. Wang and P. Lu, J. Mater. Chem. C, 2019, 7, 2430-2435.

59 K. Zhang, Y. Shen, X. Yang, J. Liu, T. Jiang, N. Finney, B. Spingler and S. Duttwyler, Chem. Eur. J., 2019, 25, 8754-8759.

60 I. Fuentes, M. J. Mostazo-Ljpez, Z. Kelemen, V. Compañ, A. Andrio, E. Moralljn, D. Cazorla-Amorjs, C. Viñas and F. Teixidor, Chem. Eur. J., 2019, 25, 14308-14319.

61 M. Jørgensen, P. T. Shea, A. W. Tomich, J. B. Varley, M. Bercx, S. Lovera, R. Černý, W. Zhou, T. J. Udovic, V. Lavallo, T. R. Jensen, B. C. Wood and V. Stavila, Chem. Mater., 2020, 32, 1475-1487.

62 M. Jørgensen, S. R. H. Jensen, T. D. Humphries, M. R. Rowles, M. V. Sofianos, C. E. Buckley, T. R. Jensen and M. Paskevicius, J. Phys. Chem. C, 2020, doi: 10.1021/acs.jpcc.0c02523;

63 Z. Li, C. Ma, Y. Wen, Z. Wei, X. Xing, J. Chu, C. Yu, K. Wang and Z. -K. Wang, Nano Research, 2020, 13, 196-202.

64 K. P. Anderson, M. A. Waddington, G. J. Balaich, J. M. Stauber, N. A. Bernier, J. R. Caram, R. I. Djurovich and A. M. Spokoyny, Dalton Trans., 2020 doi:10.1039/d0dt00826e

65 M. Keener, C. Hunt, T. G. Carroll, V. Kampel, R. Dobrovetsky, T. W. Hayton and G. Ménard, Nature, 2020, 577, 652-655. 


\section{ARTICLE}

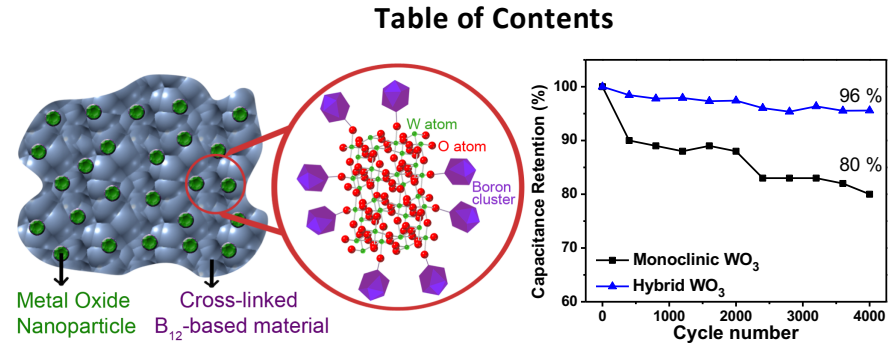

A Boron-rich hybrid tungsten oxide material with enhanced cycling stability up to 4000 cycles compared to that of pristine tungsten oxide 